\title{
A Semi-Blind Reference Video Watermarking using Hybrid Transforms for Copyright Protection
}

\author{
Satyanarayana Murty. P \\ Research scholar \\ Department of ECE \\ A.U. college of Engg(A) \\ Andhra University \\ Vishakapatnam
}

\author{
K. Venkatesh \\ UG student \\ Department of ECE \\ College of Engg, GIITS
}

\author{
Rajesh Kumar. P \\ Associate Professor \\ Department of ECE \\ A.U. college of Engg(A) \\ Andhra University \\ Vishakapatnam
}

\begin{abstract}
When network and storage devices become more popular, the digital data more easily and more quickly will be distributed. Can be easily pirated copy and did not limit a large number of multimedia information (images, audio and video). Therefore, people are increasingly concerned about the protection of intellectual property rights of multimedia. Digital watermarking is a useful and the development of the technology; it is direct information in the original additional information embedded in the technology. Ideally, the digital watermark is added to the data and the original data should be no visual difference, and the digital watermark cannot be protected information not in the case of damage easily be removed or modified. In this paper we propose a three robust and semi-blind digital video water marking algorithm. These algorithms are based on hybrid transforms using the combination of Discrete Cosine Transform (DCT) and Singular Value Decomposition (SVD), Discrete Wavelet Transform (DWT) and Singular Value Decomposition (SVD) and Discrete Cosine Transform (DCT), Discrete Wavelet Transform (DWT) and Singular Value Decomposition (SVD).The original video is divided to number of frames. On one frame we apply a correspond three hybrid transform algorithms. The process is repeated for all the reaming frames. The performance of the proposed algorithms was evaluated with respect to imperceptibility and robustness. The results show that the proposed algorithms give a good Peak Signal to Noise Ratio (PSNR), however their performance varied with respect to robustness.
\end{abstract}

\section{Keywords}

Network, Storage Devices, multimedia, watermarking, DCT, DWT, SVD, Robustness

\section{INTRODUCTION}

Due to the rapid and extensive growth of network technology, digital information can now be distributed much faster and easier. However, according to the insufficient cognizance of intellectual property, the condition of illegal copies and spread of copyright reserved information are growing serious. To protect the copyright of multimedia information and to decrease the impulse to copy and spread copy right reserved multimedia information. There are immense technical challenges in discouraging unauthorized copying and distributing of digital information. Fortunately, digital watermarking technique has been proposed as a method to embed an invisible signal into multimedia data so as to attest the owner identification of the data and discourage the unauthorized copying and distributing of digital information. In digital image watermarking the inserted watermark should not degrade the visual perception of an original image. This information of digital data can be extracted later for ownership verification [1]. Digital watermarking can applied to a variety of fields like text, image, audio, video and software. A lot of techniques are available for protecting the copyrighted material. The first method for hiding watermarking is by directly changing original cover-media. The advantages are simple and fast calculated but cannot protect itself from varied signal processing attacking [2, 3]. The most of watermarking techniques embed the information data in the coefficients of transformation domain of the cover image, such as Fourier transformation, discrete cosine transformation, wavelet transformation and singular value decomposition. Image watermarking algorithms using Discrete Cosine Transform (DCT) $[4,5,6,12,13]$, Discrete Wavelet Transform (DWT) [7, 8, 9, 10, 11], Singular Value Decomposition (SVD) $[14,15]$ are available in the literature. Domain transformation watermarking schemes, in general, first use DCT and DWT and then transforms the image into the spatial domain. Watermarking schemes usually focus on watermarking black and white or grayscale images. The data hiding capacity is high in spatial domain and frequency domain algorithms based on DCT, SVD. However, these algorithms are hardly robust against various attacks, prone to tamper and degrade the quality of the watermarked image. Hybrid domain transforms are also available in the literature DCT- SVD $[16,17,18,19]$ and DWT-SVD $[20,21,22,23,24,25,26,27,28,29,30]$. Now a day's people are looking to authenticate their video content $[31,32]$ also.

In this paper we proposed three semi - blind reference video watermarking algorithms DCT- SVD, DWT-SVD and DWTDCT-SVD. The rest of the paper is organized as follows: Section 2 describes related work, section 3 contains our proposed algorithms, while Section 4 provides experimental results and in section 5 conclusions and in Section 6 references.

\section{Spatial Frequency}

Spatial frequency of an image can be used to know the overall activity level in an image. For an image block $l_{1}$ of size $\mathrm{M} \times \mathrm{N}$, the spatial frequency is defined as:

$$
S F=\sqrt{R F^{2}+C F^{2}}
$$

Where $\mathrm{RF}$ and $\mathrm{CF}$ are the row and column frequencies and are defined as: 


$$
\begin{aligned}
& R F=\sqrt{\frac{1}{M_{1} N_{1}} \sum_{m=1}^{M 1} \sum_{N=2}^{N 1}[l(m, n)-l(m, n-1)]^{2}} \\
& C F=\sqrt{\frac{1}{M_{1} N_{1}} \sum_{N=1}^{N 1} \sum_{m=2}^{M 1}[l(m, n)-l(m-1, n)]^{2}}
\end{aligned}
$$

\section{RELATED WORK}

In this paper [33], the authors proposed two blind video watermarking algorithms based on Singular Value Decomposition transform. Initially the host video is divided into frames and on each frame they applied SVD transform. They took the binary image as a watermark to be embedded in host video frames. In the first algorithm they embed the watermark in diagonal fashion. In the second algorithm bits are inserted in a block-wise fashion. Here they consider the imperceptibility, robustness and payload of those algorithms.

In this paper [34], the authors proposed a non-blind video watermarking algorithm. Here also the total video was divided into frames and they selected randomly some frames based on some criteria. They applied Discrete Wavelet Transform to those selected frames. Now the frame divided into four sub-bands, like LL, LH, HL and HH. They consider the high frequency bands $\mathrm{LH}$ and $\mathrm{Hl}$. A Discrete Cosine transform has been applied to those high frequency sub-bands LH and HL. The mid frequency co-efficients of DCT transform were used to embed the watermark.

In this paper [35], the authors proposed a hybrid digital video watermarking using Discrete Wavelet transform and Principal Component Analysis. The video frames were decomposed into four sub-bands. Then they applied PCA to those wavelet sub-bands for watermark insertion. They used a binary image as a watermark to embed into a host video.

In this paper [36], the authors proposed a hybrid non-blind MPEG video watermark using wavelet transform and tensor algebra. They used the higher-order tensor singular value decomposition and DWT. They used the technique scene change analysis to embed the watermark.

\section{PROPOSED ALGORITHM}

The watermark embedding and extraction process has shown in figure 1 .

\subsection{Algorithm using DCT-SVD}

\section{Watermark Embedding Procedure}

The objective of this procedure is to embedded the watermark into the cover or host video without degrading the original host video.

Step 1: Divide the video scene into frames $F_{i}$,

$\mathrm{i}=1,2,3 \ldots \ldots \ldots \ldots$.
Step 2: Convert every video frame $F_{i}$ from RGB to $Y C_{b} C_{r}$ color matrix format.

Step 3: Compute the steps 4 to 11 for the $\mathrm{Y}$ matrix in each frame off $F_{i}$.

Step 4: The Y matrix is segmented into blocks of size $\mathrm{p}_{1} \times$ $\mathrm{p}_{2}$ via ZIG_ZAG sequence denoted by $\mathrm{F}^{1}$, where 1 is the number of blocks.

Step 5: Find out the spatial frequency of all blocks, denoted by $\mathrm{SF}_{\mathrm{F}} \mathrm{l}$.

Step 6: Significant blocks are found out based on their spatial frequency. Spatial frequencies of each block are stored in descending order. Then make a threshold on spatial frequency. Those blocks, which have spatial frequency less than or equal to threshold, are considered as significant blocks and are used for making reference image, $f_{r e f}$ which is a size of $m \times n$.

Step 7: Perform DCT on the reference image, which is denoted by $f_{D C T}$

Step 8: Perform SVD transform on both $f_{D C T}$ and watermark image, denoted by $f_{W}$.

$$
\begin{aligned}
& f_{d c t}^{S V D}=U_{f_{d c t}} * S_{f_{d c t}} * V_{f_{d c t}}^{T} \\
& f_{W}^{S V D}=U_{f_{W}} * S_{f_{W}} * V_{f_{W}}^{T}
\end{aligned}
$$

Step 9: Modify the single values of reference image with the singular values of watermark as

$$
\left(\sigma_{f_{r e f}}\right)^{*}=\sigma_{f_{d c t}^{S V D}}+\beta * \sigma_{f_{W}^{S V D}}
$$

Where $\beta$ give's the watermark strength.

Step 10: Perform inverse SVD,

$$
f_{i s v d}=U_{f_{d c t}} * S_{f_{r e f}}^{*} * V_{f_{d c t}}^{T}
$$

Step 11: Perform inverse DCT to construct the modified reference image, denoted by $f_{\text {idct }}$. Again $f_{\text {idct }}$ is segmented into blocks of size $p_{1} \times p_{2}$ and mapped onto their original positions for constructing the watermarked image, denoted by $F_{W}^{*}$

Step 12: Convert the video frames from $Y C_{b} C_{r}$ to RGB color matrix.

Step 13: Reconstruct frames into final watermarked video scene $F_{i}^{*}$

\section{Watermark Extraction Procedure}

The objective of the watermark extraction is to obtain the estimate of the watermark. For watermark extraction, original reference and watermarked images, left and right singular vectors must be available at the receiver end.

Step 1: Divide the watermarked video scene into frames $F_{i}^{*}$,

$\mathrm{i}=1,2,3 \ldots \ldots \ldots \ldots$.

Step 2: Convert every watermarked video frame $F_{i}^{*}$ from RGB to $Y C_{b} C_{r}$ color matrix format.

Step 3: Compute the steps 4 to 8 for the $\mathrm{Y}$ matrix in each frame of $F_{i}^{*}$. 
Step 4: Using the positions of significant blocks, make the reference image from the watermarked Y matrix, denoted by $f_{\text {ref }}^{*}$

Step 5: Perform DCT on both $f_{\text {ref }}^{*}$ and original reference image, $f_{\text {ref }}$.

Step 6: Perform SVD transform on both DCT coefficients.

$$
\left(f_{D C T}^{S V D}\right)^{*}=U_{f_{d c t}^{*}}^{*} * S_{f_{d c t}}^{*} * V_{f_{d c t}^{* T}}^{* T}
$$

$$
\sigma_{W}^{e x t}=\frac{\sigma_{f_{d c t}}^{*}-\sigma_{f_{d c t}}}{\beta}
$$

Step 8: Obtain the extracted watermark as:

$$
W^{\text {ext }}=U_{W} * S_{W}^{e x t} * V_{W}^{T}
$$

Step 9: Construct the image watermark W by cascading watermarks from all frames.

Step 7: Extract the singular values of the watermark.

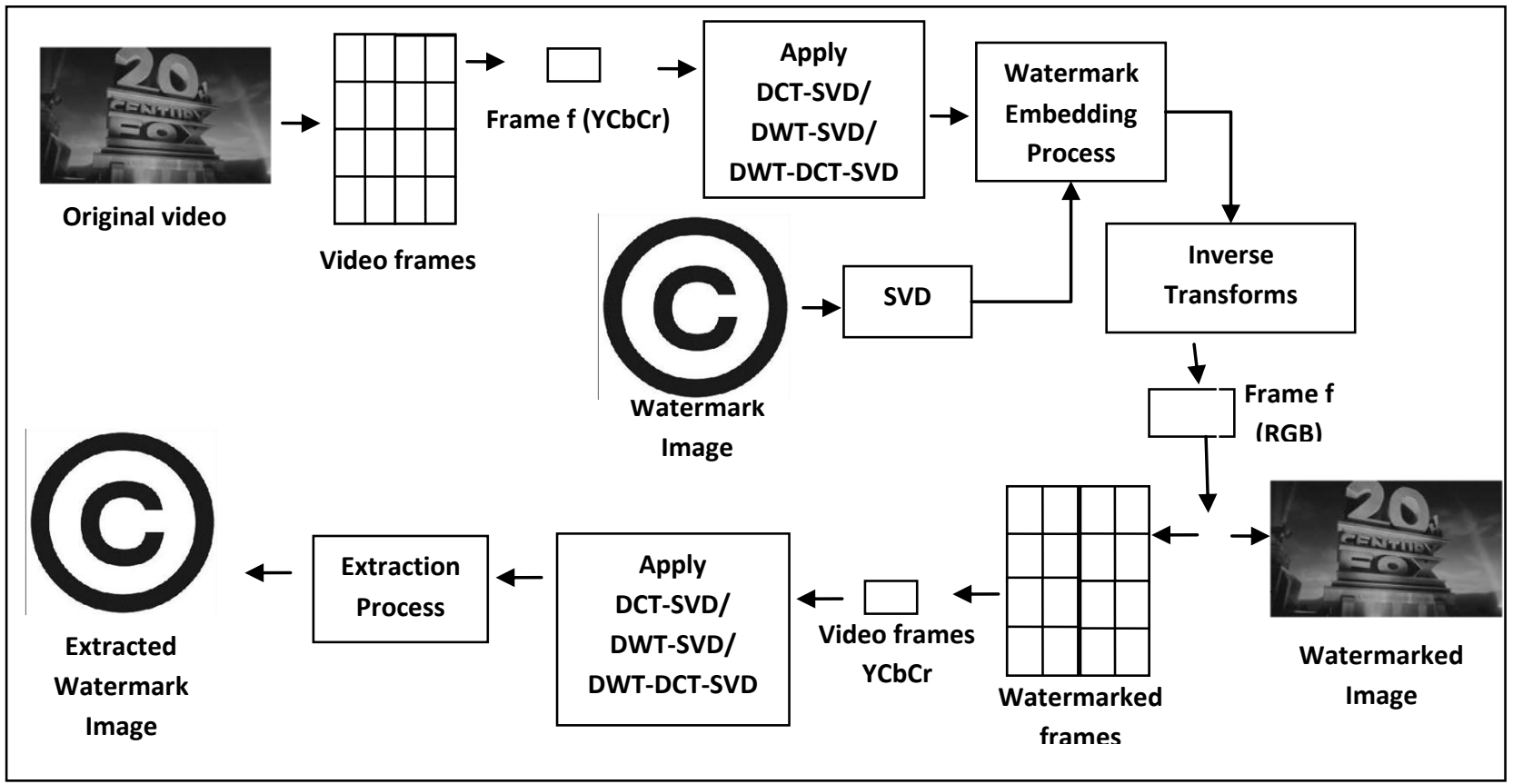

Fig 1: Generalized watermark Embedding and Extraction process

\subsection{Algorithm using DWT-SVD \\ Watermark Embedding Procedure}

Step 1: Divide the video scene into frames $F_{i}$, $\mathrm{i}=1,2,3 \ldots \ldots \ldots . . \mathrm{n}$

Step 2: Convert every video frame $F_{i \mathbb{i}}$ from RGB to $Y C_{b} C_{r}$ color matrix format.

Step 3: Compute the steps 4 to 11 for the $\mathrm{Y}$ matrix in each frame $F_{\tilde{z}}$.

Step 4: The Y matrix is segmented into blocks of size $p_{1} \times$ $\mathrm{p}_{2}$ via ZIG_ZAG sequence denoted by $\mathrm{F}^{1}$, where 1 is the number of blocks.

Step 5: Find out the spatial frequency of all blocks, denoted by $\mathrm{SF}_{\mathrm{F}} \mathrm{l}$.

Step 6: Significant blocks are found out based on their spatial frequency. Spatial frequencies of each block are stored in descending order. Then make a threshold on spatial frequency. Those blocks, which have spatial frequency less than or equal to threshold, are considered as significant blocks and are used for making reference image, $f_{\text {ref }}$ which is a size of $m \times n$
Step 7: Perform DWT on the reference image. It divides the image into four sub bands, LL, LH, HL and HH. Select the LL band, Which is denoted by $f_{L L}$.

Step 8: Perform SVD transform on both $f_{L L}$ and watermark image.

$$
\begin{aligned}
& f_{L L}^{S V D}=U_{L L} * S_{L L} * V_{L L}^{T} \\
& f_{W}^{S V D}=U_{W} * S_{W} * V_{W}^{T}
\end{aligned}
$$

Step 9: Modify the single values of reference image with the singular values of watermark as

$$
\left(\sigma_{f_{\text {ref }}}\right)^{*}=\sigma_{L L}+\beta * \sigma_{W}
$$

Here $\beta$ give's the watermark strength.

Step 10: Perform inverse SVD,

$$
f_{i S V D}^{*}=U_{L L} * S_{f_{r e f}}^{*} * V_{L L}^{T}
$$

Step 11: Perform inverse DWT to construct the modified reference image, denoted by $f_{D W T}^{*}$. Again $f_{D W T}^{*}$ is segmented into blocks of size $\mathrm{p}_{1} \times \mathrm{p}_{2}$ and mapped onto their original positions for constructing the watermarked image. 
Step 12: Convert the video frames from $Y C_{b} C_{r}$ to RGB color matrix.

Step 13: Reconstruct frames into final watermarked video scene $F_{\mathrm{i}}^{*}$

\section{Watermark Extraction Procedure}

Step 1: Divide the watermarked video scene into frames $F_{i}^{*}$, $\mathrm{i}=1,2,3 \ldots \ldots \ldots \ldots$.

Step 2: Convert every watermarked video frame $F_{i}^{*}$ from RGB to $Y C_{b} C_{r}$ color matrix format.

Step 3: Compute the steps 4 to 8 for the $\mathrm{Y}$ matrix in each frame $F_{i}^{*}$.

Step 4: Using the positions of significant blocks, makethe reference image from the watermarked Y matrix, $f_{\text {ref }}^{*}$.

Step 5: Perform DWT on both $\mathrm{f}_{\text {ref }}^{*}$ and original reference image, $f_{\text {ref }}$.

Step 6: Perform SVD transform on both $f_{L L}$ and $f_{\text {ref }}^{W}$.

$$
\left(f_{L L}^{S V D}\right)^{*}=U_{L L}^{*} * S_{L L}^{*} * V_{L L}^{* T}
$$

Step 7: Extract the singular values of the watermark.

$$
\sigma_{W}^{e x t}=\frac{\sigma_{L L}^{*}-\sigma_{f_{L L}}}{\beta}
$$

Step 8: Obtain the extracted watermark as:

$$
W^{\text {ext }}=U_{W} * S_{W}^{e x t} * V_{W}^{T}
$$

Step 9: Construct the image watermark $W$ by cascading watermarks from all frames.

\subsection{Algorithm using DWT- DCT-SVD}

Watermark Embedding Procedure

Step 1: Divide the video scene into frames $F_{i}$,

$\mathrm{i}=1,2,3 \ldots \ldots \ldots \ldots$.

Step 2: Convert every video frame $F_{i}$ from $\mathrm{RGB}$ to $Y C_{b} C_{r}$ color matrix format.

Step 3: Compute the steps 4 to 11 for the $\mathrm{Y}$ matrix in each frame $F_{i}$.

Step 4: The Y matrix is segmented into blocks of size $\mathrm{p}_{1} \times$ $\mathrm{p}_{2}$ via ZIG_ZAG sequence denoted by $\mathrm{F}^{1}$, where 1 is the number of blocks.

Step 5: Find out the spatial frequency of all blocks, denoted by $\mathrm{SF}_{\mathrm{F}} \mathrm{l}$.

Step 6: Significant blocks are found out based on their spatial frequency. Spatial frequencies of each block are stored in descending order. Then make a threshold on spatial frequency. Those blocks, which have spatial frequency less than or equal to threshold, are considered as significant blocks and are used for making reference image, $f_{r e f}$ which is a size of $m \times n$
Step 7: Perform DWT on the reference image. It divides the image into four sub bands, LL, LH, HL and HH. Select the LL band, which is denoted by $f_{L L}$. Perform DCT on the $f_{L L}$, which is denoted by $f_{D C T}^{L L}$.

Step 8: Perform SVD transform on both $c$ and watermark image, $\mathrm{W}$.

$$
\begin{gathered}
f_{D C T}^{L L, S V D}=U_{f_{d c t}} * S_{f_{d c t}} * V_{f_{d c t}}^{T} \\
W=U_{W} * S_{W} * V_{W}^{T}
\end{gathered}
$$

Step 9: Modify the single values of reference image with the singular values of watermark as

$$
\left(\sigma_{f_{r e f}}\right)^{*}=\sigma_{f_{d c t}}+\beta * \sigma_{W}
$$

Where $\beta$ give's the watermark strength.

Step 10: Perform inverse SVD,

$$
f_{i S V D}^{*}=U_{f_{d c t}} * S_{f_{d c t}}^{*} * V_{f_{d c t}}^{T}
$$

Step 11: Perform inverse DCT denoted by $f_{i D C T}^{*}$. Now perform DWT on $f_{i D C T}^{*}$, which is denoted by $f_{i D W T \text {. and is }}^{*}$ segmented into blocks of size $\mathrm{p}_{1} \times \mathrm{p}_{2}$ and mapped onto their original positions for constructing the watermarked image.

Step 12: Convert the video frames from $Y C_{b} C_{r}$ to RGB color matrix.

Step 13: Reconstruct frames into final watermarked video scene $F_{\mathrm{i}}^{*}$.

\section{Watermark Extraction Procedure}

Step 1: Divide the watermarked video scene into frames $F_{\mathrm{i}}^{*}$, where $i=1,2,3 \ldots \ldots \ldots \ldots$.

Step 2: Convert every watermarked video frame $F_{i}^{*}$ from RGB to $Y C_{b} C_{r}$ color matrix format.

Step 3: Compute the steps 4 to 8 for the $\mathrm{Y}$ matrix in each frame $F_{i}^{*}$.

Step 4: Using the positions of significant blocks, make the reference image from the watermarked Y matrix , $f_{r e f}^{*}$

Step 5: Perform DWT and DCT on both $f_{r e f}^{*}$ and original reference image, $f_{\text {ref }}$.

Step 6: Perform SVD transform on both DCT coefficients.

$$
f_{\text {ref }}^{W}=U_{f_{\text {ref }}^{W}} * S_{f_{\text {ref }}^{W}} * V_{f_{\text {ref }}^{W}}^{T}
$$

Step 7: Extract the singular values of the watermark.

$$
\sigma_{W}^{e x t}=\frac{\sigma_{f_{r e f}^{W}}-\sigma_{f_{d c t}}}{\beta}
$$

Step 8: Obtain the extracted watermark as:

$$
W^{\text {ext }}=U_{W} * S_{W}^{e x t} * V_{W}^{T}
$$

Step 9: Construct the image watermark $W$ by cascading watermarks from all frames. 


\section{EXPERIMENTAL RESULTS}

We evaluated the performance of proposed video watermarking algorithms with host video clips of size xxx. The video clip is divided into number of frames. The watermark used in our experiment was a gray scale image. Snapshot from the video and the watermark are shown in Figure 2 (a) and (b) respectively.

\subsection{Imperceptibility Performance}

Imperceptibility means that the perceived quality of the video clip should not be distorted by the presence of the watermark [37]. As a measure of the quality of a watermarked video, the peak signal to noise ratio (PSNR) is typically used has shown in equation 26. In our work, the watermark was embedded in the video according to the algorithms discussed in the section 3. For the algorithm DCT-SVD the embedding depth of watermark was 0.02. The corresponding average PSNR for the all frames of the watermarked video was $41.6962 \mathrm{db}$. We used the watermark depth for DWT-SVD and DWT-DCT-SVD was 0.035. The corresponding PSNR values were 42,2704db and $41.1233 \mathrm{db}$ respectively.

$$
\begin{aligned}
& R M S E=\sqrt{\frac{[f(i, j)-F(i, j)]^{2}}{N^{2}}} \\
& P S N R=20 \log \frac{255}{R M S E} d b
\end{aligned}
$$

RMSE Is the Root Mean Square Error and is a comparison between the host image and watermarked image.

$f(i, j)$ and $F(i, j)$ represent host and watermarked images respectively. Size of the host image is $\mathrm{N} \mathrm{x} \mathrm{N}$.

\subsection{Robustness Performance}

Robustness of a watermarking algorithm is a measure of the immunity or resistance of the watermark against attempts to remove or degrade it from the video frames by different types of digital signal processing attacks [38]. The similarity between the original watermark and the extracted watermark from the attacked watermarked video frames was measured by using the correlation factor $\rho$, which is computed using the following Equation:

$$
\rho(w, \widetilde{w})=\frac{\sum_{i=1}^{N} w_{i} \widetilde{w}_{i}}{\sqrt{\sum_{i=1}^{N} w_{i}^{2}} \sqrt{\sum_{i=1}^{N} \widetilde{w}_{i}^{2}}}
$$

Where $\mathrm{N}$ is the number of pixels in watermark, $\mathrm{w}$ and $\tilde{w}$ is the original and extracted watermarks respectively. The correlation factor $\rho$, may take values between -1 and 1 .

We evaluated robustness of the algorithm against the following video attacks: JPEG compression, video frame rotation, noise attacks (Gaussian and salt \& pepper), cropping, resize, histogram equalization, motion blur, average and median filtering.
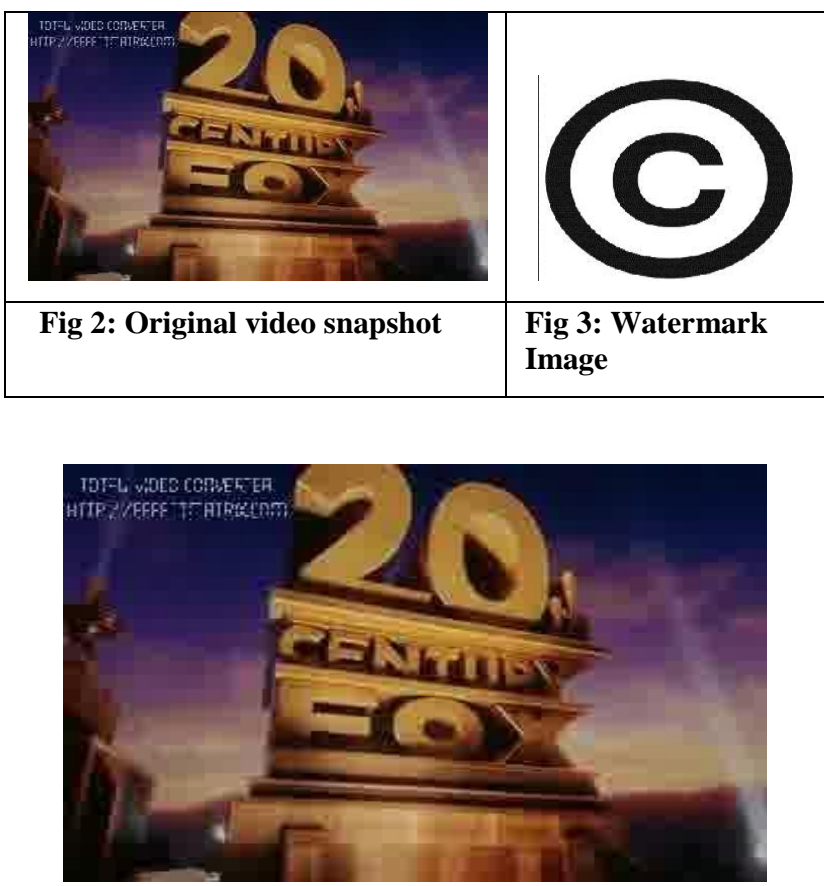

Fig 4: watermarked video snapshot

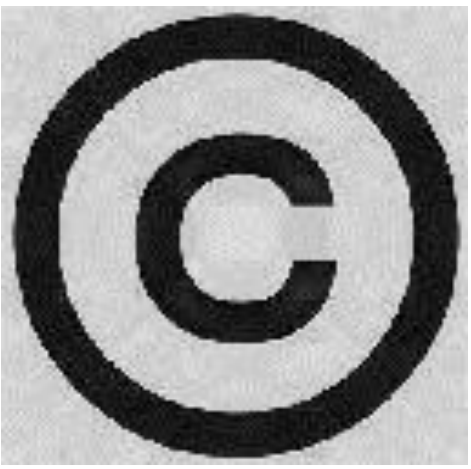

Fig 5: Extracted watermarked

\subsection{ATTACKS}

\subsubsection{JPEG compression}

The watermarked video frames were compressed with different quality factors. As shown in figure 6 the correlation values indicate clearly the high robustness of the proposed algorithms. DWT-DCT-SVD method has shown high robustness when compared with other two methods. But at 20 and 54 compression ratios the DWT-SVD was showing good robustness. 


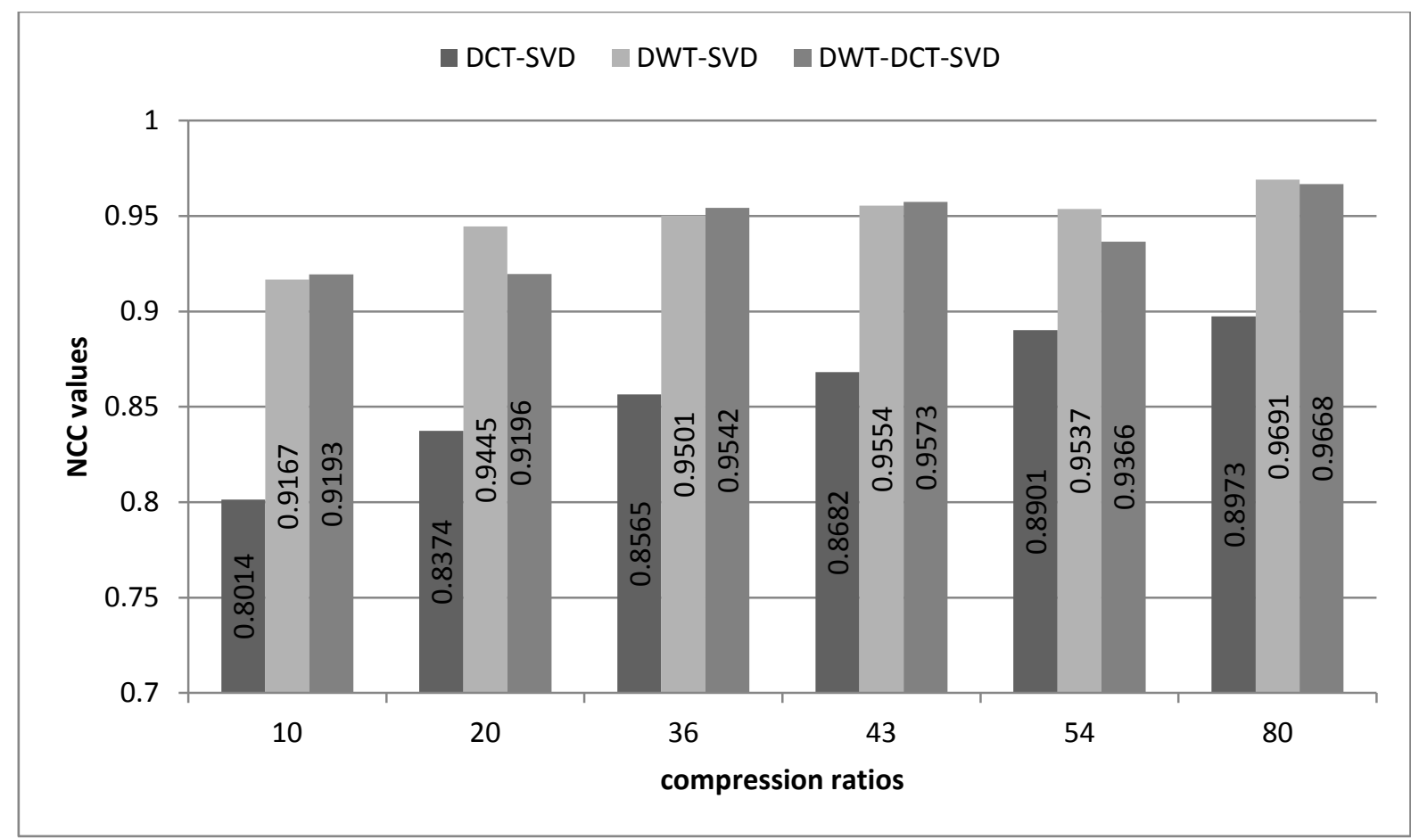

Fig 6: compression attack

\subsubsection{Video Frame Rotation}

The watermarked video frames were rotated with different angles. As shown in figure 7. The correlation values indicate robustness of the algorithms against the video frames rotation. The DCT-SVD algorithm has shown good robustness against other algorithms.

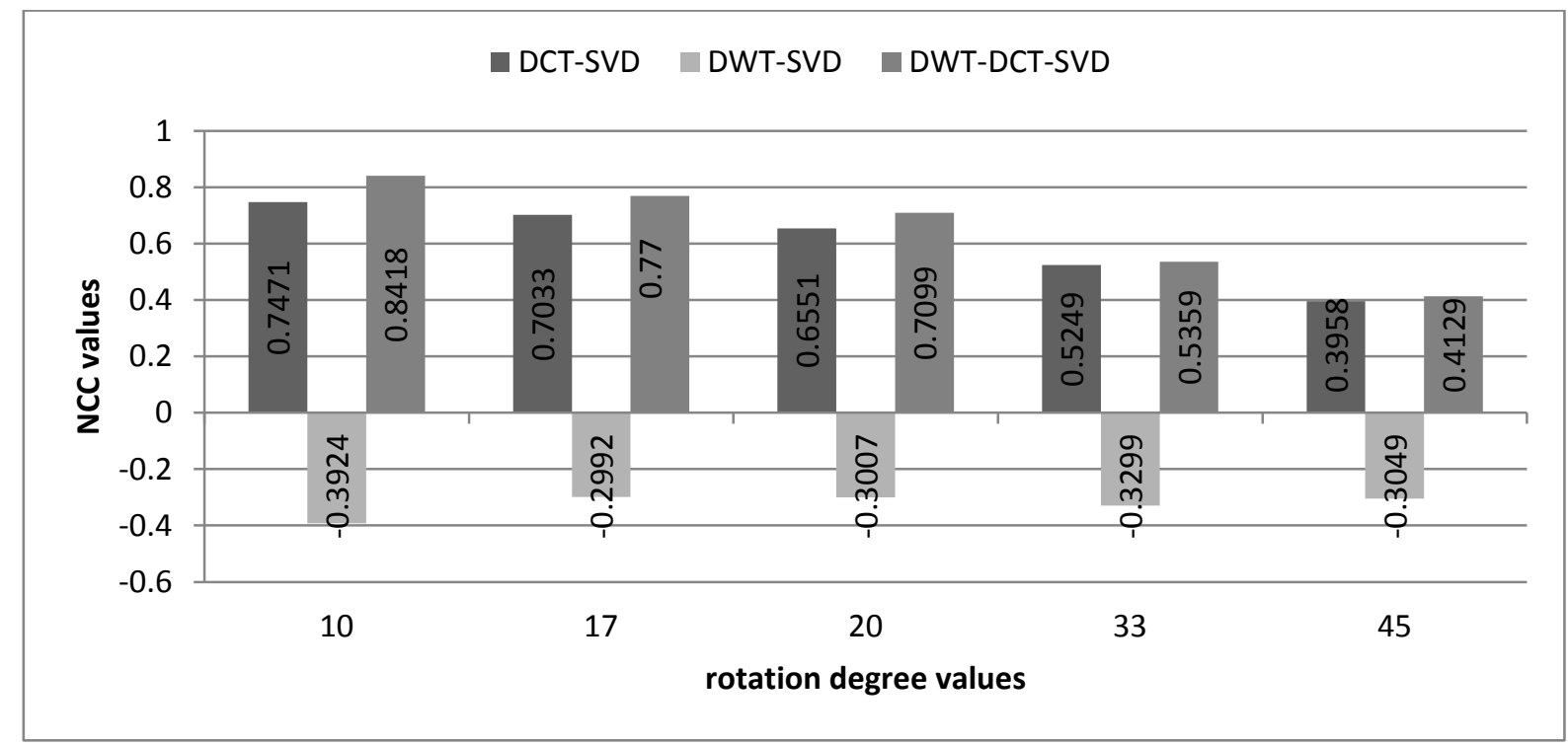

Fig 7: Rotation attack

\subsubsection{Additive Gaussian and Salt \& Pepper}

Two kinds of common attacks were Additive Gaussian, Salt \& pepper noise. Each noise was tested separately with varying intensities to the watermarked video frames. As shown in Figure8 and figure 9respectively. These results generally indicate robustness of the proposed algorithms against addition of Gaussian and Salt and Pepper noise. The DWT - SVD algorithm has shown high robustness against other two algorithms. But at $75 \%$ of Salt \& Pepper noise the DCT-SVD has shown good robustness. 


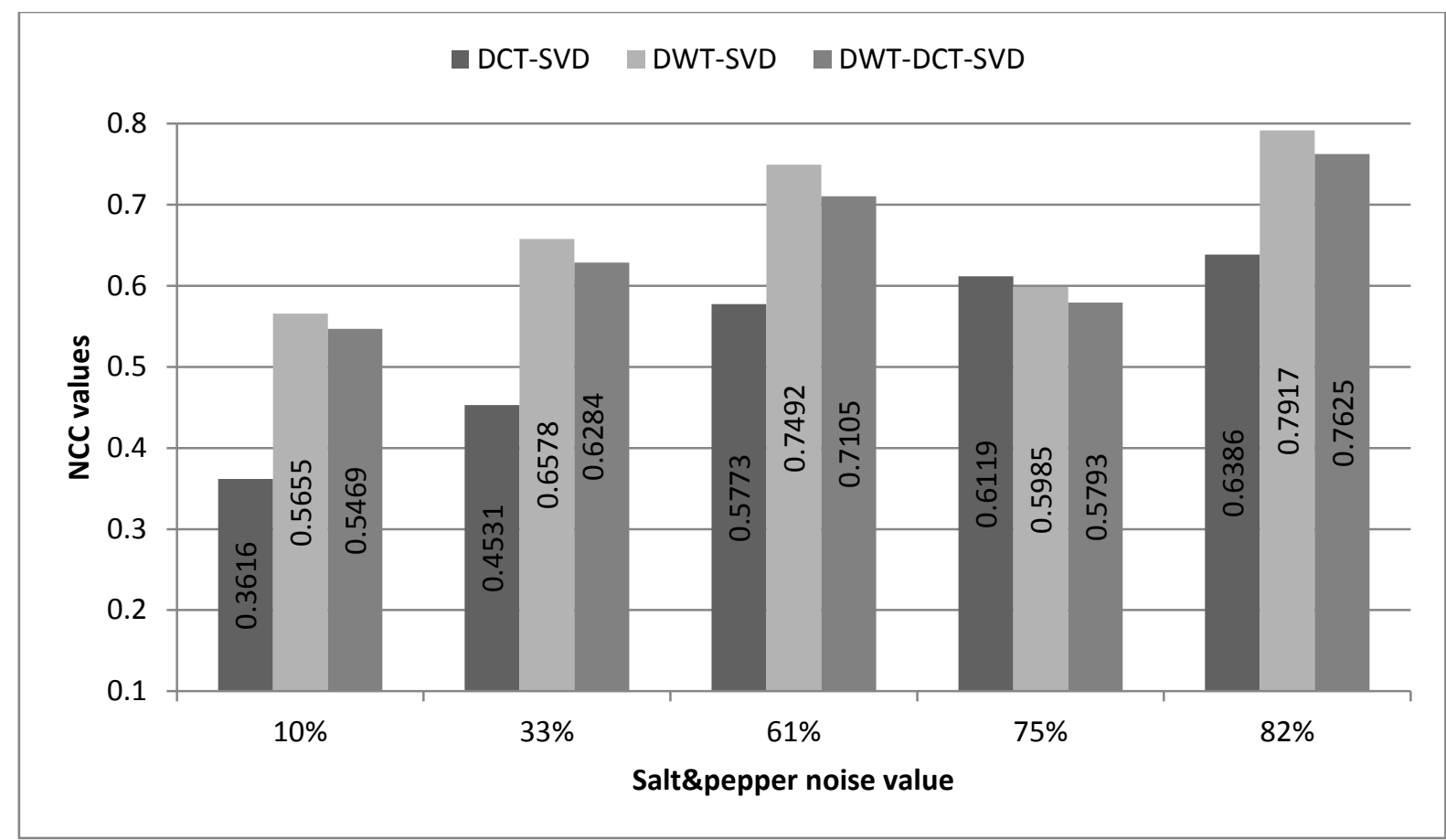

Fig 8: Salt \& Pepper attack

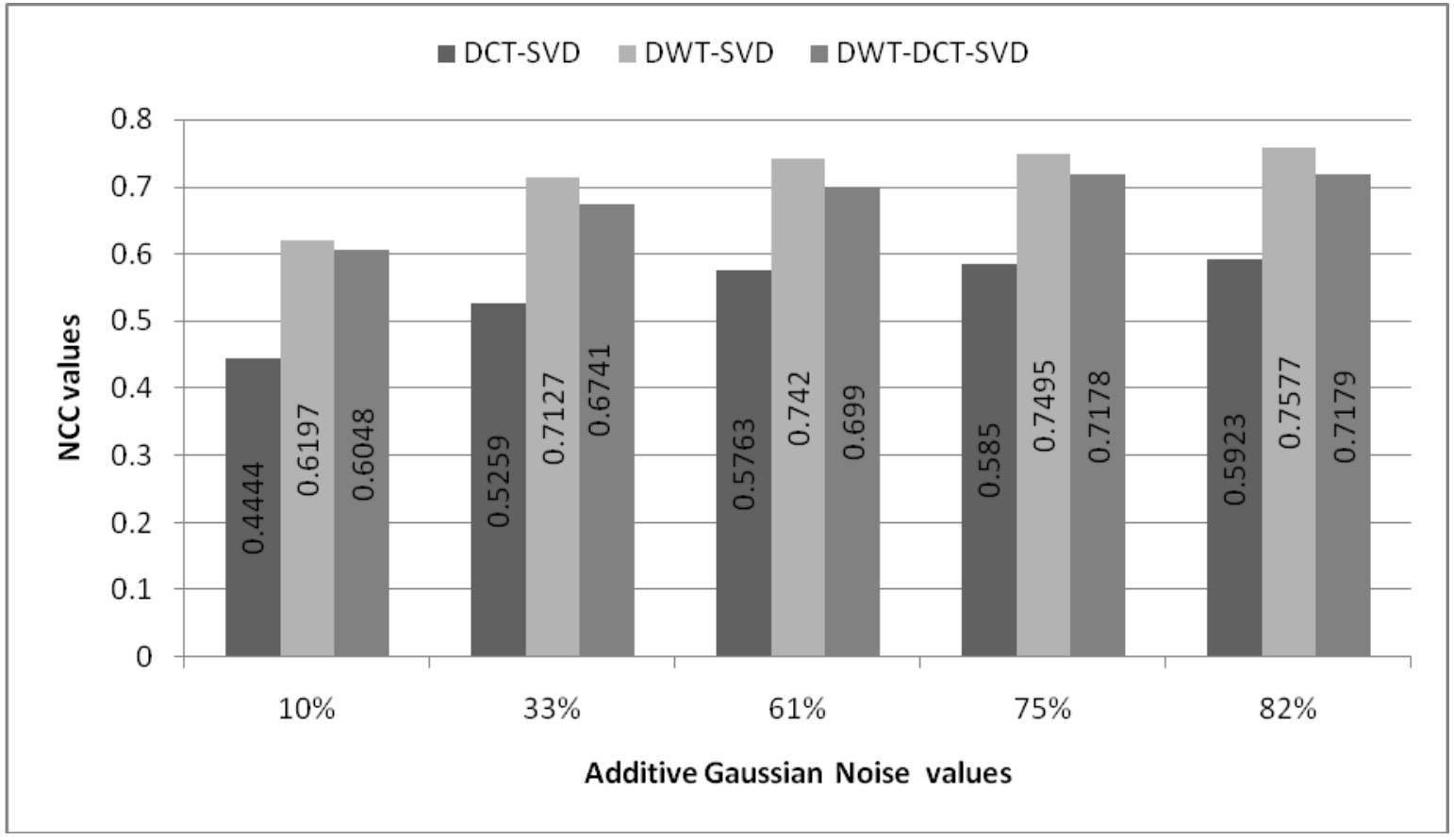

Fig 9: Additive Gaussian Noise attack

\subsubsection{Average filtering and Median filtering}

Other important attacks on video were average filtering and median filtering. We tried to mask the video by different mask values. This has sown in figure 10 and figure 11 respectively. We got good robustness values for these masks. For average filtering and median filtering DWT-DCT-SVD algorithm has good robustness values against other two algorithms. 


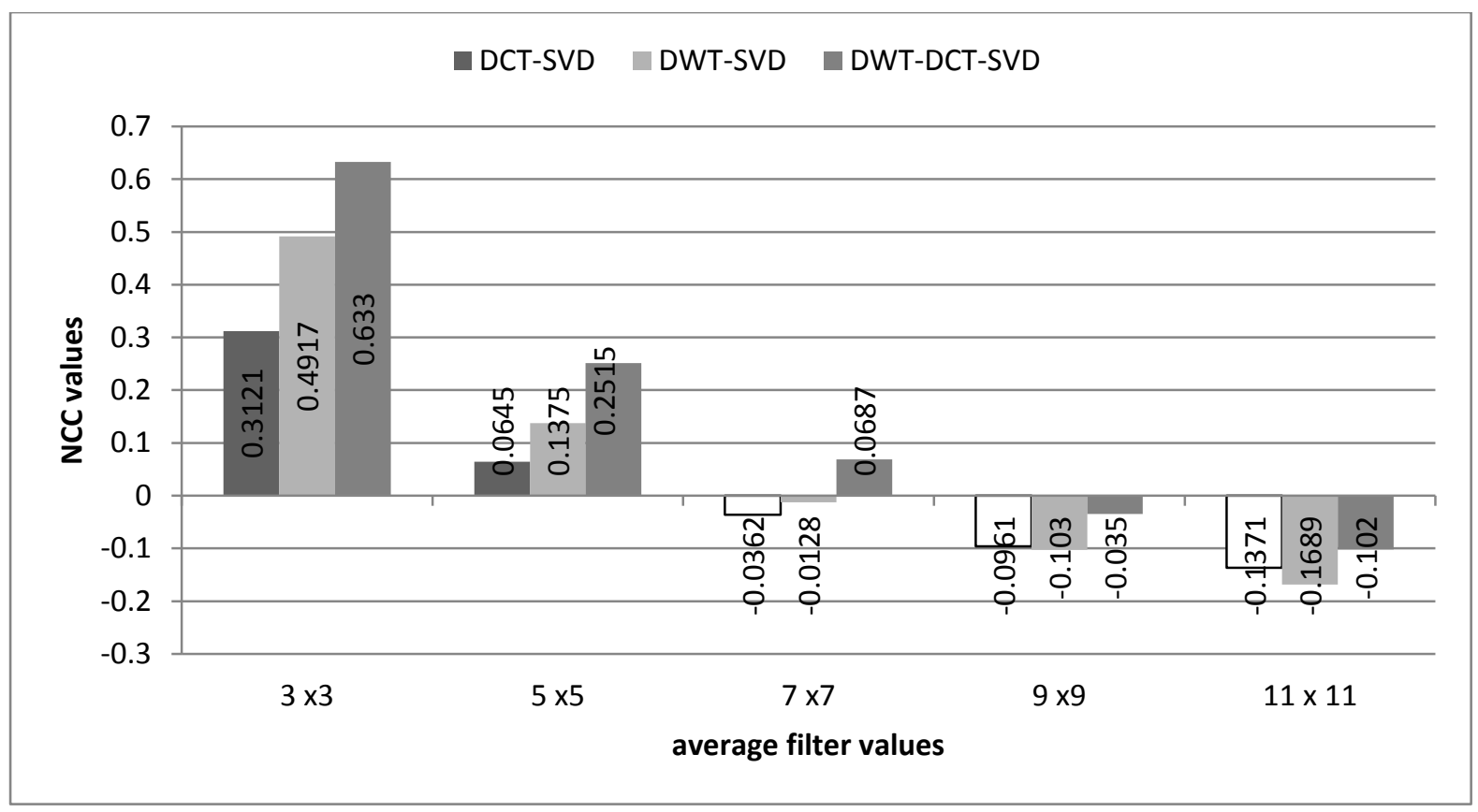

Fig 10: Average filtering attack

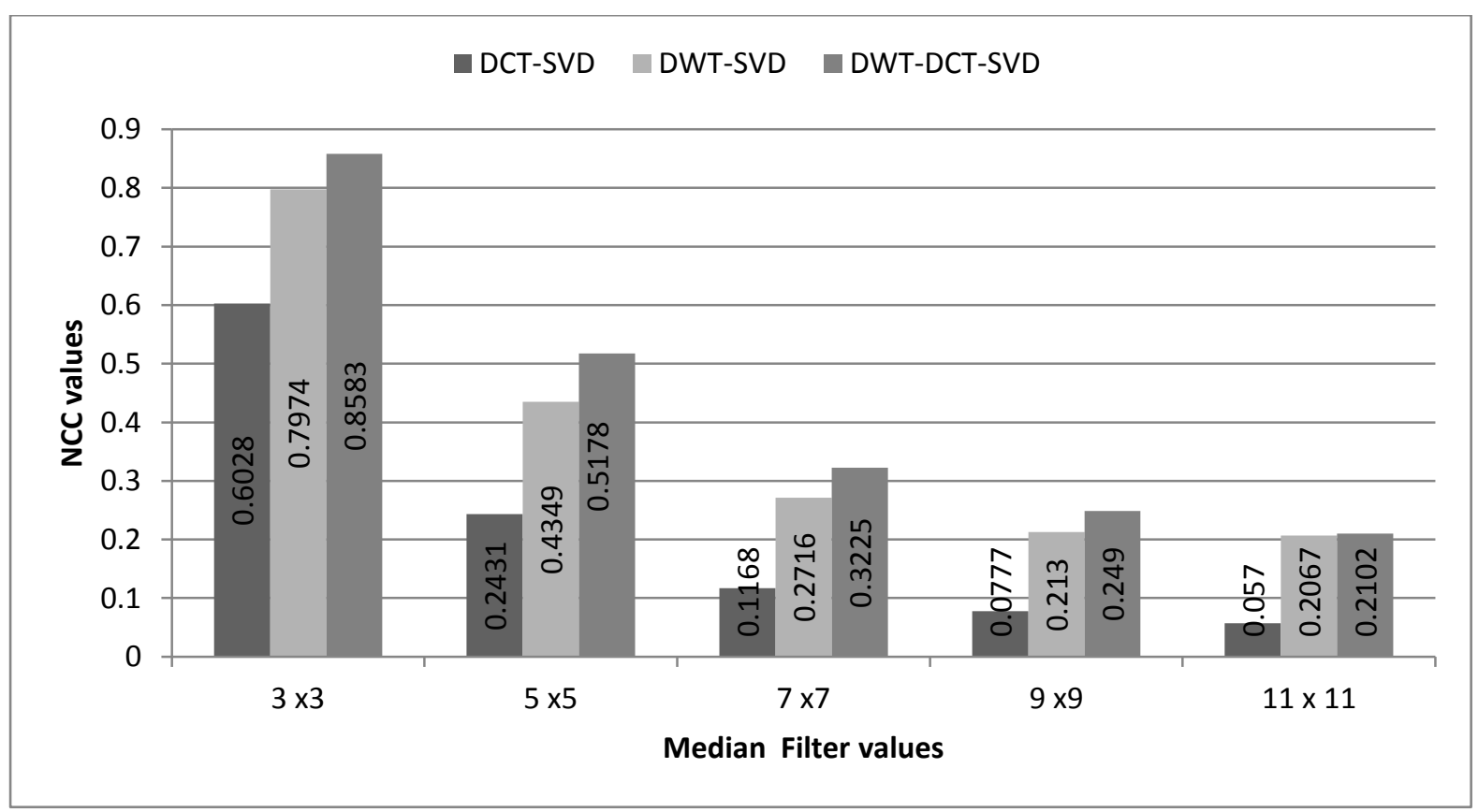

Fig 11: Median Filter attack

\subsubsection{Cropping and resizing}

Another important attack was cropping. Here we cropped the video such that $25 \%$ area was reaming. It showed that DWTDCT-SVD algorithm was good robustness against other two algorithms. The other prime image processing attack was resizing. The resizing was performed from 512-> 128-> 512 . The DWT-DCT-SVD algorithm showed high robustness against other two algorithms. The robustness values of cropping and resizing were shown in figure 12 (a) and figure 12 (b) respectively. 


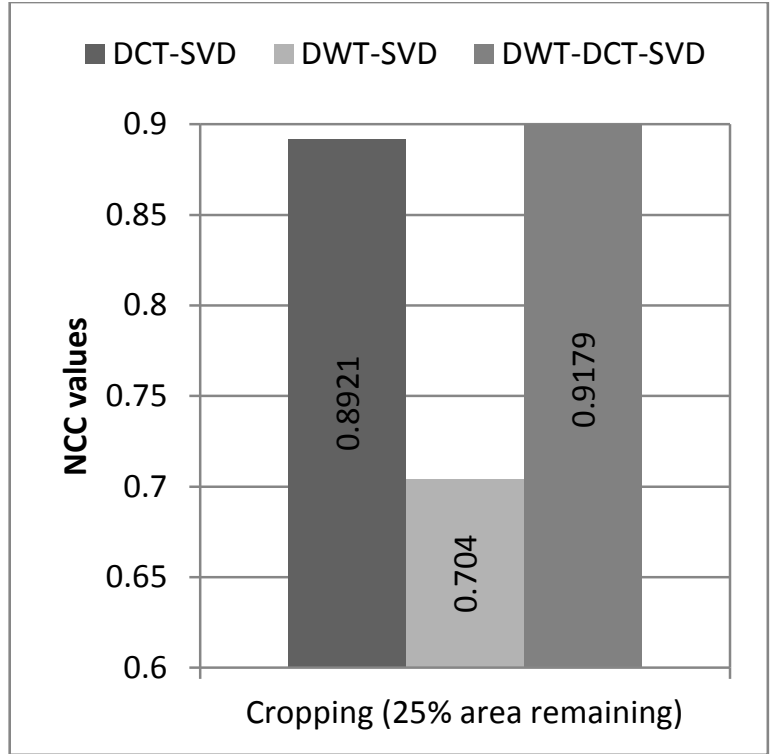

(a) Cropping

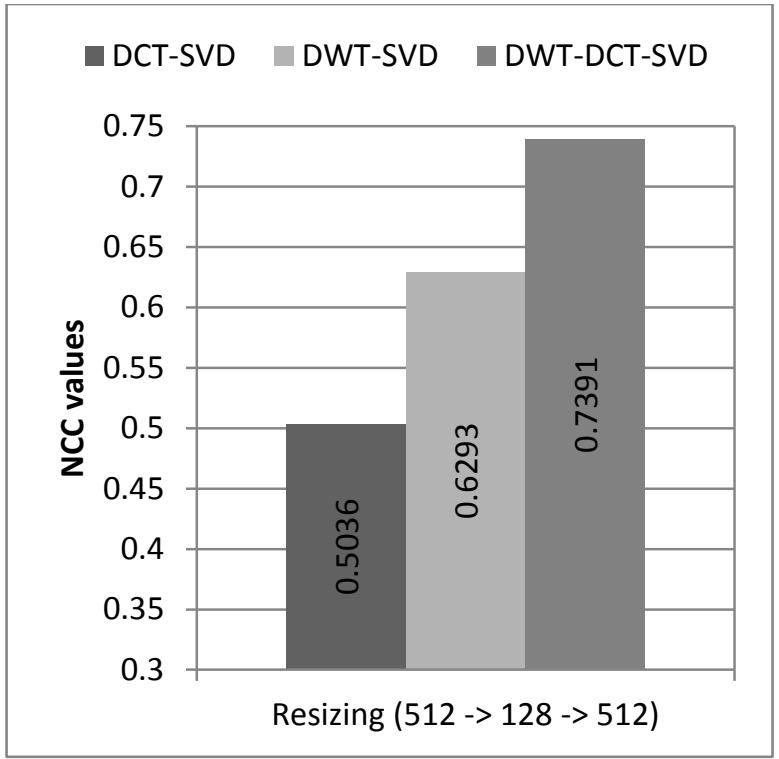

(b) Resizing

Fig 12: Cropping and resizing attacks

\subsubsection{Histogram equalization and Motion blur}

Histogram equalization and motion blur attacks were shown in figure 13. It showed that the proposed algorithms had high robustness against these attacks. For both the attacks the DWT-DCT-SVD algorithm provided good robustness than other

two algorithms.
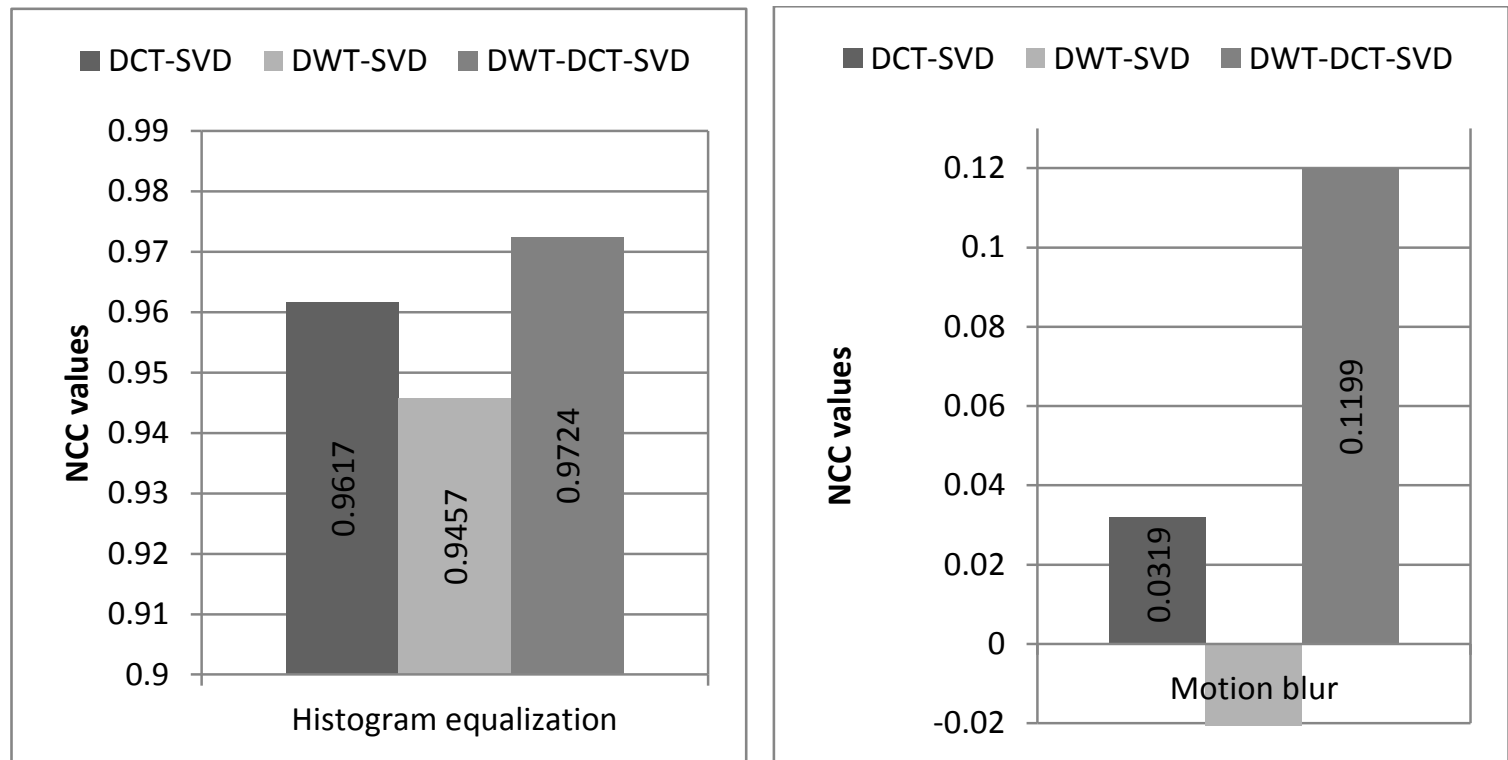

Fig 12: Histogram Equalization and Motion Blur attacks

\section{CONCLUSION}

In this paper we proposed three semi-blind reference watermarking algorithms. Those algorithms were DCT-SVD, DWT-SVD and DWT-DCT-SVD. The watermark was visually meaningful gray scale image. In these proposed algorithms DWT-SVD has good imperceptibility. When considered the robustness of these algorithms the DWT-DCTSVD algorithm was good. Except one attack, ie Salt \&
Pepper noise, the algorithms DWT-SVD and DWT-DCTSVD were superior to the DCT-SVD algorithm. Out of these algorithms, the hybrid algorithm DWT-DCT-SVD has optimum good imperceptibility and robustness at various attacks. When we consider the payload of these algorithms the DCT-SVD has high payload or high capacity of watermark embedding. 


\section{REFERENCES}

[1] V.Potdar, S. Han, and E. Chang, 2005. "A Survey of Digital Image Watermarking Techniques", in Proceedings of the 2005 IEEE International Conference on Industrial Informatics, pp. 709-716.

[2] F. Hartung and M. Kutter, "Multimedia Watermarking Techniques," in Proceedings of the IEEE, vol. 87, no.7, pp. 1079-1107, July 1999

[03].Chi-Kwong Chan , L.M. Cheng "Hiding data in images by simple LSB substitution "Pattern

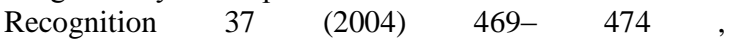
ww.elsevier.com/locate/patcog

[4]. Dr.M.A.Dorairangaswamy "A Robust Blind Image Watermarking Scheme in Spatial Domain for Copyright Protection" International Journal of Engineering and Technology Vol. 1, No.3, August, 2009 , ISSN: 1793-8236

[5] I. Pitas,"A method for signature casting on digital images,"Proceedings of IEEE International Conference on Image Processing,” Vol. 3, pp.215-318 ,1996.

[6]. R. Wolfgang and E. Delp, "A watermark for digital images," Proceeding of IEEE International Conference on Image Processing, Vol. 2, pp.319-222, 1996.

[7]. Sanghyun Joo, Youngho Suh, Jaeho Shin, and Hisakazu Kikuchi "A New Robust Watermark Embedding into Wavelet DC Components " ETRI Journal, Volume 24, Number 5, October 2002.

[8]. Sadi Vural, Hiromi Tomii, Hironori Yamauchi "DWT Based Robust Watermarking Embed Using CRC-32 Techniques" World Academy of Science, Engineering and Technology 52005.

[9]. Kundur D., and Hatzinakos, D., 'A Robust Digital Image Water- marking Scheme using WaveletBased fusion,' Proc. IEEE Int. Conf. On Image Processing, Santa Barbara, California, vol. 1, pp. 544547, October 1997.

[10].V. Padmanabha Reddy and Dr. S. Varadarajan “ An Effective Wavelet-Based Watermarking Scheme Using Human Visual System for Protecting Copyrights of Digital Images "International Journal of Computer and Electrical Engineering, Vol. 2, No. 1, February, 2010 ,pp1793-8163.

[11].X. Xia, C.G. Boncelet, and G.R. Arce, "A Multiresolution Watermark for Digital Images," Proc. Of IEEE ICIP, Santa Barbara, CA, USA, Oct. 1997, pp. 548-551.

[12].J.R. Kim and Y.S. Moon, "A Robust WaveletBased Digital Watermarking Using evel-Adaptive Thresholding," Proc. Of IEEE ICIP, vol. 2, Kobe, Japan, Oct. 1999, pp. 226-230.

[13].S. D. Lin and C.-F. Chen,"A Robust DCT-Based Watermarking for Copyright Protection," IEEE Transactions on Consumer Electronics, 46(3), August 2000, pp. 415-421.

[14].Gengming Zhu, and Nong Sang "Watermarking Algorithm Research and Implementation Based on DCT Block " proceedings of world academy of science, engineering and technology volume 35 november 2008 issn 2070-3740 .

[15].H. Andrews and C. Patterson, "Singular Value decompositions and Digital Image Processing," IEEE Transactions on Acoustics, Speech and Signal Processing, vol. 24, no. 1, pp. 26-53, Feb. 1976.

[16].Ke-feng He, "Watermarking for images using the HVSand SVD in the wavelet domain" Proceedings of IEEEInternational Conference on Mechatronics andAutomation, pp. 2352- 2356, 2006.

[17].Liu Quan, and AI Qingsong "A combination of DCT based and SVD based watermarking," ICSPproceedings of IEEE International conference on signalprocessing, pp. 873-876, 2004

[18].Alexander Sverdlov, Scott Dexter, Ahmet M. Eskicioglu "Robust SVD DCT based watermarking for copyright protection", IEEE Transactions on Image Processing, 10(5), May 2001, pp. 724-735.

[19].Feng Liu, and Yangguang Liu, "A Watermarking Algorithm for Digital Image Based on DCT and SVD,"Congress on Image and Signal Processing, pp. 380-383, 2008

[20].Liu Liang and Sun Qi, "A new SVD-DWT composite watermarking," ICSP proceedings of IEEE International conference on signal processing, 2006.

[21].Gaurav Bhatnagar and Balasubramanian Raman, "Anew robust reference watermarking scheme based onDWTSVD," Computer Standards and Interfaces, vol.31, no. 5, pp. 1002-1013, 2009.

[22].E.Ganic and A.M. Eskiciogulu et.al., Robust embedding of Visual Watermarks using DWT-SVD Journal of Electronic Imaging, October-December, 2005.

[23].Q. Li, C. Yuan, and Y. Zhong, "Adaptive DWT-SVD Domain Image Watermarking Using Human Visual Model," proceedings of 9th international conference on advanced communication Technology, vol. 3, pp. 19471951, Feb.2007.

[24]. Ali Al-Haj, and T. Manasrah, "Non-Invertible Copyright Protection of Digital Images Using DWTand SVD," Proceedings of International Conference on Digital Information Management, vol. 1, pp. 448 - 453,2007.

[25].S. Rezazadeh, and M. Rahmati, "A robust watermarking scheme based on wavelet transform and block SVD," 9th International Symposium on Signal Processing and Its Applications, pp. 1-4, 2007.

[26].Swanirbhar Majumder, Madhusudhan Mishra, A Dinamani Singh" A Hybrid SVD and Wavelet based Watermarking "2nd National Conference Mathematical Techniques: Emerging Paradigms for Electronics and IT Industries, September 26-28, 2008, pp. 197-205.

[27].Humberto Ochoa , K.R. Rao “A Hybrid DWT-SVD Image-Coding System (HDWTSVD) for Color Images "systemics, cybernetics and informatics volume 1 . number 2, pp.64-70.

[28].T.Sridevi, Y.Ramadevi,V.Vijaya Kumar "SECURE WATERMARKING BASED ON SVD AND WAVELETS "ICGST-GVIP Journal, Volume 10, Issue 5, December 2010.

[29].Satyanarayana Murty . P, M.Uday Bhaskar, P.Nanna 
Babu, P. Rajesh Kumar "A Semi-Blind Reference Watermarking Scheme Using DWT-SVD for Copyright Protection "The International Journal of Multimedia \& Its Applications (IJMA) Vol.3, No.3,pp.61-70.

[30]. Saryanarayana Murty.

$P$,

Laxmi.V , Rajesh Kumar.P "Performance of Semi-Blind Reference Watermarking Scheme Using DWT-SVD for Copyright Protection" (IJCSIT) International Journal of Computer Science and Information Technologies, Vol. 3 (2), 2012,3321-3327

[31].Langelaar, G., I. Setyawan, and R. Lagendijk, 2000. "Watermarking Digital Image and Video Data: A Stateof-Art Overview", IEEE Signal Processing Magazine 17, pp. 20-46.

[32].D. Kundur, K. Su and D. Hatzinakos, 2004. "Digital Video Watermarking: Techniques, Technology and Trends", in Intelligent Watermarking Techniques, Chapter 10, P. J.-S. Pan, H.-C. Huang and L. Jain, eds., World Scientific Publishing Company. pp. 265-314.

[33].Lama Rajab, Tahani Al-Khatib, Ali Al-Haj "Video Watermarking Algorithms Using the SVD Transform" European Journal of Scientific Research ISSN 1450216X Vol.30 No.3 (2009), pp.389-401.

[34].Aditi Agarwal , Ruchika Bhadana and Satishkumar Chavan, "A Robust Video Watermarking Scheme using DWT and DCT" (IJCSIT) International Journal of Computer Science and Information Technologies, Vol. 2 (4) , 2011, 1711-1716.

[35]. Sanjana Sinha, Prajnat Bardhan, Swarnali Pramanick, "Digital Video Watermarking using Discrete Wavelet Transform and Principal Component Analysis" International Journal of Wisdom Based Computing, Vol. 1 (2), August 2011.

[36].Emad E. Abdallah , A. Ben Hamza , Prabir Bhattacharya "Video watermarking using wavelet transform and tensor algebra" Published online: 24 April 2009 (C) SpringerVerlag London Limited 2009.
[37]. Voloshynovskiy, S., S. Pereira, and T. Pun, 2001. "Attacks on Digital Watermarks: Classification,Estimation-Based Attacks, and Benchmarks". Communications Magazine 39, pp.118126.

[38].Fabien A.P. Petitcolas, Ross J. Anderson, 1999. "Evaluation of Copyright Marking Systems", IEEE International Conference on Multimedia Computing and Systems (ICMCS'99) 1, pp. 574-579.

\section{AUTHOR'S PROFILE}

P. Satyanarayana Murty is currently working as Sr.Associate Professor in ECE Department, GIITS, Engineering College, Vishakapatnam, and Andhra Pradesh, India. $\mathrm{He}$ is working towards his Ph.D.at AU College of Engineering, Vishakapatnam, India. He received his M.Tech from Jawaharlal Nehru Technological University, Hyderabad, India. He has fifteen years experience of teaching undergraduate students and post graduate students. His research interests are in the areas of image watermarking, and image compression

Dr. P. Rajesh Kumar is currently Associate Professor in ECE Department, AU College of Engineering, Vishakapatnam, India. He received his M.Tech and Ph.D. from AndhraUniversity, Vishakapatnam, India. He has eleven years experience of teaching undergraduate and postgraduate students and guided number of post-graduate theses. He has published 10 research papers in National and International journals. Presently he is guiding six Ph.D students in the area of digital signal processing and Image processing. His research interests are in the areas of digital image processing and digital signal processing. 